\title{
High-rate altimetry in SNR-based GNSS-R: Proof-of-concept of a synthetic vertical array
}

\author{
M. K. Yamawaki, ${ }^{1}$ F. Geremia-Nievinski, ${ }^{2}$ J. F. Galera Monico ${ }^{3}$
}

\begin{abstract}
Global Navigation Satellite System Reflectometry (GNSS-R) has emerged as a promising remote sensing technique for coastal sea level monitoring. The GNSS-R based on signal-tonoise ratio (SNR) observations employs a single antenna and a conventional receiver. It performs best for low elevation satellites, where direct and reflected radio waves are very similar in polarization and direction of arrival. One of the disadvantages of SNR-based GNSS-R for sea level altimetry is its low temporal resolution, which is of the order of one hour for each independent satellite pass. Here we present a proof-of-concept based on a synthetic vertical array. It exploits the mechanical movement of a single antenna at high rate (about $1 \mathrm{~Hz}$ ). SNR observations can then be fit to a known modulation, of the order of the antenna sweeping rate. We demonstrate that centimetric altimetry precision can be achieved in a 5-minute session.
\end{abstract}

Index Terms-GNSS; GNSS Reflectometry;

\section{INTRODUCTION}

Coastal regions are vulnerable to sea level rise and frequent extreme events. For climate change mitigation and adaptation, it is fundamental to monitor sea surface height variations [1]. Unfortunately, conventional tide gauges are not as numerous as necessary. This is because they require a supporting structure very close to the water, which is costly to install and maintain, and not even feasible at more challenging coast lines [2]. Furthermore, tide gauges require geodetic monitoring for vertical land motion control [3].

Global Navigation Satellite System Reflectometry (GNSS-R) is a promising alternative for coastal sea level altimetry [4] . One of the pioneering works was [5], who employed signal-tonoise ratio (SNR) observations. It demonstrated the feasibility of measuring sea level via the interference pattern between direct and reflected ray paths. SNR-based GNSS-R has better response at low elevation satellites, where the multiple ray paths have similar polarization and direction of arrival [6]. Furthermore, as sensing occurs at slant incidence, sea surface altimetry is feasible from a sensor installed away from the water [7]

Unfortunately, SNR-based GNSS-R needs approximately one hour per altimetry retrieval, as it relies on satellite elevation angle variations. The low retrieval rate is one of the main

Paper submitted on 08/31/2020 for review. This work was funded by the National Council for Scientific and Technological Development (CNPq) grants number 372541/2013-5, 457530/2014-6, and 433099/2018-6, as well as by the Rio Grande do Sul State Research Funding Agency (Fapergs) grant number 26228.414.42497.26062017

M. K. Yamawaki: Postgraduate Program in Remote Sensing, Federal University of Rio Grande do Sul (UFRGS), RS, 91509-900, Brazil; (kenji.yamawaki@gmail.com).

F. Geremia-Nievinski: Department of Geodesy and Postgraduate Program in Remote Sensing, Federal University of Rio Grande do Sul, RS, 91509-900, Brazil; (e-mail: felipe.nievinski@ufrgs.br)

J. F. Galera Monico: Department of Cartography and Postgraduate Program in Cartographic Sciences, São Paulo State University, SP, 19060900, Brazil; (e-mail: galera.monico@unesp.br). weakness of this technique [5]. Decreasing the period between altimetry retrievals has the potential to improve the monitoring of fast-changing extreme events, such as tide storm surges [8], [9].

To improve the temporal resolution, [10] introduced a calibration step, displacing the GNSS antenna vertically by means of a manually operated rope and pulley assembly. They were thus able to retrieve reflector height with centimeter level accuracy within 10 minutes for a single satellite. It is also possible to combine multiple simultaneous satellites through a filter for improved resolution [11]. Another study [12] was able to retrieve the GNSS antenna vibration frequency by analyzing SNR readings. In GNSS positioning, it is possible to mitigate multipath via randomization by subjecting the antenna to high frequency movement [13]-[16].

Here we demonstrate a proof-of-concept of a synthetic vertical array for GNSS-R. By vertically displacing a single GNSS antenna in a sweeping fashion, spatial diversity can be leveraged for high-rate altimetry. The antenna displacement allows us to synthesize an antenna array, as if we had several real antennas aligned vertically. This is not unlike the use of forward motion in a synthetic aperture radar.

SNR-based GNSS-R employing a single static antenna can take about 1 hour to retrieve reflector height. In contrast, we can perform altimetry within 5 minutes. Below we describe the Materials and Methods; Experiment Setup; Results and Discussion. We end the study with conclusions and recommendations for future work.

\section{MAterials AND MethodS}

In the following subsections, we describe the mathematical models applied for modeling the data and the electromechanical device design and assembly. Before field experiment, we tested the proposed methodology on SNR data obtained on an open source GNSS multipath simulator [17]. This way we could determinate beforehand the optimal setup for the field experiment and some expected results.

\section{A. Forward Model}

SNR-based GNSS-R starts with raw SNR data, $S$ (in linear power scale) being detrended [18]: 


$$
s(t)=S(t)-\bar{S}(t)
$$

where the SNR trend, $\bar{S}$, is a low order polynomial stemming from the antenna gain pattern. Detrended SNR, $s$, is then fit to a damped sinusoid of the form:

$$
s(t)=A_{S} \cos (\phi)
$$

It involves the SNR oscillation amplitude $A_{S}$ (in watts per watt) and the interferometric phase $\phi=\phi_{r}-\phi_{d}$, the difference between reflected and direct phases.

Normally a static planar reflecting surface is assumed, $\phi \cong$ $\Delta \phi$, producing:

$$
\Delta \phi=k_{z} H+\phi_{0},
$$

where $H$ is the antenna height above the surface (the reflector depth), typically unknown; $k_{z}=2 k \sin (e)$ is the so-called vertical wavenumber, in terms of the ordinary wavenumber $k=$ $2 \pi / \lambda$, where $\lambda$ is the wavelength (all known quantities); and $\phi_{0}$ is a phase shift. The satellite elevation angle $e$ changes slowly with time, so its temporal dependence is postponed for section II.D. This standard model is appropriate for altimetry applications; it neglects a smaller phase component stemming from the antenna pattern and the surface material [18].

In the proposed method, the static phase model above $\Delta \phi$ caused by the slow satellite movement is complemented by a dynamic phase variation $\delta \phi$ of instrumental origin:

$$
\phi(t)=\Delta \phi+\delta \phi(t)
$$

This new fast-changing term $\delta \phi$ has a known functional form, based on the sensor construction. Here we postulate a sinusoidal phase variation over time,

$$
\delta \phi(t)=A_{\phi} \cos \left(2 \pi f \cdot t+\varphi_{0}\right)
$$

where $A_{\phi}$ is the phase amplitude (in radians), $f$ is the sweeping frequency (in hertz), and $\varphi_{0}$ is another arbitrary phase-shift. Detrended SNR will thus read:

$$
s(t)=A_{S} \cos (\Delta \phi+\delta \phi(t))
$$

It should be noted that although the dynamic phase term $\delta \phi$ has a sinusoidal form, the corresponding variation induced in SNR is no longer a damped sinusoid, as the cosine of a cosine is a non-trivial function.

\section{B. Electromechanical Apparatus}

The dynamic phase variation $\delta \phi=k_{z} \delta H$ arises from the antenna vertical displacement $\delta H=A_{H} \cos \left(2 \pi f t+\varphi_{0}\right)$, with $A_{H}$ being the antenna sweeping amplitude (in meters). It gets projected in the interferometric geometry implied by the vertical wavenumber $k_{z}$. For example, for a satellite at zenith, $\delta \phi$ is simply the round-trip distance between antenna and surface, expressed in radians; for a low-elevation satellite, the phase amplitude will be near zero, meaning that the vertical displacement is not effective for a satellite near the horizon.

The sinusoidal vertical displacement is achieved in practice with the electromechanical apparatus shown in Fig. 1 and Fig. 2. A scotch Yoke converts angular motion into linear motion by means of a slotted link mechanism. A 12-V DC electric motor drives the mechanical system at a given sweeping frequency $f$ of the order of $1 \mathrm{~Hz}$. The sweeping phase shift $\varphi_{0}$ is related to the starting position of the mechanism. The sweeping amplitude $A_{H}$ (in meters) is basically the lever arm length, of the order of a decimeter.
Ideally the parameters related to the apparatus design and construction would be calibrated based on external measurements. For example, an a priori value of $A_{H}$ may be obtained simply with a ruler or tape measurement. The sweeping phase shift $\varphi_{0}$ is more difficult to determine but it should remain constant for each time series.

\section{SNR Fitting}

SNR is sampled multiple times per sweeping cycle. The resulting time series is partitioned in $N$ short-duration nonoverlapping sets, each lasting for about one sweeping cycle, $\left\{S_{i}^{j}\right\}$, where the indices are $i=1,2, \ldots, N$ for each cycle and $j=1,2, \ldots, M_{i}$ for each measurement in the $i$-th set. As the sweeping period is short enough, the satellite elevation angle $e_{i}^{j} \approx e_{i}$ can be approximated as piece-wise constant, and in fact so can the whole static phase component $\Delta \phi_{i}^{j} \approx \Delta \phi_{i}$.

SNR can then be fit via non-linear least squares as follows:

$$
\begin{gathered}
S_{i}^{j}(t)=\bar{S}_{i}+A_{S i} \cos \left(\Delta \phi_{i}+\delta \phi_{i}^{j}(t)\right) \\
\delta \phi_{i}^{j}(t)=k_{z i} A_{H} \cos \left(2 \pi f t_{i}^{j}+\varphi_{0 i}\right)
\end{gathered}
$$

The independent variables are time, $t_{i}^{j}$, recorded as a data time stamp, and vertical wavenumber, $k_{z i}=2 k \sin e_{i}$, where the elevation angle is interpolated from satellite ephemeris. The mechanical sweeping frequency $(f)$ and amplitude $\left(A_{H}\right)$ are regarded as known values. The unknown parameters involved in SNR fitting are:

- $\quad$ static phase $\left(\Delta \phi_{i}[\mathrm{rad}]\right)$

- $\quad$ sweeping phase shift $\left(\varphi_{0 i}[\mathrm{rad}]\right)$

- $\quad S N R$ trend $\left(\bar{S}_{i}[\mathrm{~W} / \mathrm{W}]\right)$

- $\quad S N R$ amplitude $\left(A_{S i}[\mathrm{~W} / \mathrm{W}]\right)$

Notice the SNR trend $\bar{S}_{i}^{j} \approx \bar{S}_{i}$ and SNR amplitude $A_{S i}^{j} \approx A_{S i}$ are here approximated as piece-wise constant values.

\section{Altimetry Retrieval}

Repeating the SNR fitting above yields a series of independent static phase values $\Delta \phi_{i}$. The altimetry retrieval is then achieved via a simple linear regression of $\Delta \phi_{i}$ on $k_{z i}$ (eq.3):

$$
\left[H, \phi_{0}\right]=\operatorname{argmin}\left(\sum\left|\Delta \phi_{i}-k_{z i} H-\phi_{0}\right|^{2}\right)
$$

The regression intercept is the phase-shift $\phi_{0}$ and the regression slope is the surface height $H$, which is the main quantity of interest. We assume the satellite elevation angle has changed sufficiently during the regression period.

\section{EXPERIMENT SETUP}

The experiment was carried out on January 30, 2014 at latitude $-22.12257^{\circ}$ and longitude $-51.4078^{\circ}$. The antenna was setup upright (boresight facing zenith) on top of a 1.2-meter concrete pillar in a green field (Fig. 3). There was moderate clearance to the horizon (Fig. 4); some quadrants were compromised by trees and buildings below $10^{\circ}$ elevation.

We have utilized a Trimble R9 GNSS receiver to record SNR at 50-hertz sampling rate. A Septentrio PolaNt-x MF antenna was coupled to the upper end of the Scotch yoke device (Fig. 1). The sweeping frequency was about $0.75 \mathrm{~Hz}(\sim 1.3 \mathrm{~s}$ period $)$ and the sweeping amplitude was $6.5 \mathrm{~cm}$. Those values were verified via post-processed kinematic positioning relative to a base station over a short 300-meter baseline (not shown). Of the 
total session duration (20 minutes), we selected a 5-minute portion during which the sweeping frequency was most stable.

For altimetry validation, we performed an optical survey of the site terrain around the antenna. A total station (theodolite with laser distance meter) was setup on the same pillar and 70 points were measured radially at several azimuths (Fig. 3). A planar surface was fit to points, resulting in a slight tilting $\left(2.1^{\circ}\right)$ facing northeast $\left(53.3^{\circ}\right.$ aspect angle); the root-mean-square error of the fit was $10.3 \mathrm{~cm}$.

\section{RESUltS AND DisCUSSIONS}

We focus on two GLONASS satellites that had the best observation conditions: R02 due northeast and R24 due southeast. The satellite elevation angles could not be too high, otherwise the first Fresnel zone would contain the pillar, or the antenna gain pattern would suppress the reflections. Conversely, the elevation could not be too low, otherwise the surface reflections would leave the green field, or the satellite line of sight could be blocked by clutter in the horizon.

Previous GNSS-R studies have concluded that GLONASS SNR observations have less noise [19]. That may explain the better quality of R02 and R24, in comparison to GPS satellites at similar azimuth and elevation. Other satellites, such as G05 and R01, did not have enough variation in elevation angle. The altimetry retrieval is obtained from the regression slope of the reflection phase-shift versus vertical wavenumber (Fig. 7). As the latter is related directly to the sine of elevation angle, a satellite that remains at a fixed elevation cannot be used in SNR-based GNSS-R, regardless of the tracking time.

As stated in section III, we selected the best 5 minutes of the entire 20 minutes session. This was necessary to guarantee the sweep frequency stability. As described in section II-C (eq. 7), the sweeping frequency is considered a known value, so a significant drift may hamper the SNR fitting solution. This drift was primarily caused by the motor's draining battery and fatigue of the mechanical parts (Scotch yoke).

Fig. 5 shows the SNR measurements recorded for satellite R02 in the L2 carrier and civil modulation. The high-frequency SNR oscillations, stemming from the intentional antenna sweeping, produce a few-dB wide envelope in the SNR curve. To facilitate the visualization, we show in Fig. 6 a zoom on just a few cycles. It can be noticed that SNR measurements have quarter-dB numerical resolution and near sinusoidal shape.

The SNR curve fitting is shown superimposed to SNR measurements in Fig. 6. Each cycle produces an independent static phase estimate $\Delta \phi_{i}$, depicted as black dots in Fig. 7. Despite some phase noise, a linear trend is readily noticed. The regression line is superimposed on the experimental phase estimates in Fig. 7. As explained in section II-D, the regression slope amounts to the altimetry retrieval.

For satellites R02 and R24, we obtained respectively $H=$ $1.412 \mathrm{~m}$ and $H=1.570 \mathrm{~m}$. The corresponding terrain height evaluated at different azimuths based on the field survey (Table I), yields absolute errors of $7.7 \mathrm{~cm}$ and $11.5 \mathrm{~cm}$, respectively. These centimetric values are of the same order of magnitude as the surface fitting RMSE reported at the end of section III. Besides genuine non-planar surface deviations, other possible sources of systematic error are atmospheric refraction [16] as well as the antenna pattern and the surface material composition
[18]. It is remarkable that we could perform altimetry in about 5 minutes with a near-surface SNR-based GNSS-R sensor. Fig. 8 shows a simulation for a moving antenna (black line) and a static antenna (red line). The simulation setup had similar satellite elevation and time interval as for the field experiment data of satellite R02 (Fig. 5). For a static antenna, this time interval is insufficient to retrieve reflector height, due to the lack of at least one full SNR oscillation cycle. With a moving antenna, we could expedite the reflector height retrieval, thus significantly improving the temporal resolution of SNR-based GNSS-R altimetry.

\section{CONCLUSIONS AND Future WORK}

SNR-based GNSS-R is a proven remote sensing method for coastal sea altimetry. One of its main weaknesses is the low temporal resolution, which is dependent on the satellite movement in the sky. We demonstrated a proof-of-concept for improving the temporal resolution by means of a synthetic vertical array, displacing a single antenna to exploit spatial diversity. Altimetry results were of centimetric accuracy for a 5-minute session, which is reasonable given the terrain at the site and other neglected biases.

For future work, we recommend choosing a site with fewer obstructions of the horizon around the antenna. We also expect better results using a smaller antenna, with less multipath mitigation, allowing deeper interference nulls. The power supply of the Scotch-yoke mechanism should also be revisited, to avoid sweeping frequency drift with battery power depletion.

In addition to altimetry, the high-rate retrievals demonstrated here could be helpful for the calibration of antennas installed at GNSS-R tracking stations and for the investigation of systematic effects, such as atmospheric delays [20].

\section{ACKNOWLEDGMENTS}

We thank Italo Tsuchiya for helping with the device assembly and Matheus Ferreira e Silva for the topographical surveying, both from São Paulo State University, campus Presidente Prudente.

\section{REFERENCES}

[1] O. Pörtner et al., "Special Report on the Ocean and Cryosphere in a Changing Climate." 2019, [Online]. Available: https://www.ipcc.ch/srocc/chapter/technicalsummary/.

[2] Intergovernmental Oceanographic Commission, "Manual on sea level measurement and interpretation, v. V: Radar gauges." UNESCO, 2016, [Online]. Available: https://unesdoc.unesco.org/ark:/48223/pf0000246981.

[3] P. Woodworth, G. Wöppelmann, M. Marcos, M. Gravelle, and R. Bingley, "Why We Must Tie Satellite Positioning to Tide Gauge Data," Eos, Jan. 2017, doi: 10.1029/2017EO064037.

[4] F. Geremia-Nievinski et al., "SNR-based GNSS reflectometry for coastal sea-level altimetry - Results from the first IAG inter-comparison campaign," Journal of Geodesy, 2020, doi: 10.1007/s00190-020-01387-3.

[5] K. D. Anderson, "Determination of water level and tides using interferometric observations of GPS signals," 
Journal of Atmospheric and Oceanic Technology, vol. 17, no. 8, pp. 1118-1127, Aug. 2000, doi: 10.1175/15200426(2000)017<1118:DOWLAT>2.0.CO;2.

[6] F. G. Nievinski and K. M. Larson, "Forward modeling of GPS multipath for near-surface reflectometry and positioning applications," GPS Solutions, vol. 18, no. 2, pp. 309-322, Apr. 2014, doi: 10.1007/s10291-013-0331$\mathrm{y}$.

[7] M. A. R. Fagundes, I. Mendonça-Tinti, A. L. Iescheck, D. M. Akos, and F. Geremia-Nievinski, "An open-source low-cost sensor for SNR-based GNSS reflectometry: Design and long-term validation towards sea level altimetry," GPS Solutions (accepted), p. 20, 2021.

[8] D. Peng, E. M. Hill, L. Li, A. D. Switzer, and K. M. Larson, "Application of GNSS interferometric reflectometry for detecting storm surges," GPS Solut, vol. 23, no. 2, p. 47, Mar. 2019, doi: 10.1007/s10291019-0838-y.

[9] P. L. Vu et al., "Identifying 2010 Xynthia Storm signature in GNSS-R-based tide records," Remote Sensing, vol. 11, no. 7, p. 782, Apr. 2019, doi: 10.3390/rs11070782.

[10] M. Ribot et al., "Normalized GNSS Interference Pattern Technique for Altimetry," Sensors, vol. 14, no. 6, pp. 10234-10257, Jun. 2014, doi: 10.3390/s140610234.

[11] J. Strandberg, T. Hobiger, and R. Haas, "Real-time sealevel monitoring using Kalman filtering of GNSS-R data," GPS Solut, vol. 23, no. 3, p. 61, Apr. 2019, doi: 10.1007/s10291-019-0851-1.

[12] I. Peppa, P. Psimoulis, and X. Meng, "Modelling antenna vibrations using the Signal-to-Noise Ratio (SNR) of GNSS signals," presented at the Proceedings of the 4th Joint International Symposium on Deformation Monitoring (JISDM), Athens, Greece, May 2019.

[13] B. J. H. van den Brekel and D. J. R. van Nee, "GPS multipath mitigation by antenna movements," Electronics Letters, vol. 28, no. 25, pp. 2286-2288, Dec. 1992, doi: 10.1049/el:19921471.

[14] T. Pany and B. Eissfeller, "Demonstration of a Synthetic Phased Array Antenna for Carrier/Code Multipath Mitigation," in Proceedings of the 21st International Technical Meeting of the Satellite Division of The Institute of Navigation (ION GNSS 2008), Savannah, GA, Sep. 2008, pp. 663-668.

[15] T. Ertan, M. L. Psiaki, B. W. O’Hanlon, R. A. Merluzzi, and S. P. Powell, "GNSS Multipath Mitigation using High- Frequency Antenna Motion," in Proceedings of the 26th International Technical Meeting of the Satellite Division of The Institute of Navigation (ION GNSS+ 2013), Nashville, TN, Sep. 2013, pp. 154-175.

[16] J. Wang and K. Zhang, "Low-cost positioning with rotating antenna in constrained environment for global navigation satellite systems," Electronics Letters, vol. 54, no. 1, pp. 45-47, Jan. 2018, doi: 10.1049/el.2017.3381.

[17] F. G. Nievinski and K. M. Larson, "An open source GPS multipath simulator in Matlab/Octave," GPS Solutions, vol. 18, no. 3, pp. 473-481, Jul. 2014, doi: 10.1007/s10291-014-0370-z.
[18] F. G. Nievinski and K. M. Larson, "Inverse Modeling of GPS Multipath for Snow Depth Estimation-Part I: Formulation and Simulations," IEEE Transactions on Geoscience and Remote Sensing, vol. 52, pp. 6555-6563, 2014, doi: 10.1109/TGRS.2013.2297681.

[19] S. Tabibi, F. G. Nievinski, and T. van Dam, "Statistical Comparison and Combination of GPS, GLONASS, and Multi-GNSS Multipath Reflectometry Applied to Snow Depth Retrieval," IEEE Transactions on Geoscience and Remote Sensing, vol. 55, no. 7, pp. 3773-3785, Jul. 2017, doi: 10.1109/TGRS.2017.2679899.

[20] S. D. P. Williams and F. G. Nievinski, "Tropospheric delays in ground-based GNSS multipath reflectometryExperimental evidence from coastal sites," Journal of Geophysical Research: Solid Earth, vol. 122, no. 3, pp. 2310-2327, 2017, doi: 10.1002/2016JB013612.

TABLE I

FULL RESULTS FOR TWO SATELLITES.

\begin{tabular}{cccccc}
\hline \hline $\begin{array}{c}\text { Satellite } \\
\text { ID }\end{array}$ & $\begin{array}{c}\text { Satellite } \\
\text { elevation }\end{array}$ & $\begin{array}{c}\text { Satellite } \\
\text { azimuth }\end{array}$ & $\begin{array}{c}\text { GNSS-R } \\
\text { height }\end{array}$ & $\begin{array}{c}\text { Terrain } \\
\text { height }\end{array}$ & $\begin{array}{c}\text { Height } \\
\text { error }\end{array}$ \\
\hline R02 & $18.73^{\circ}$ & $66.05^{\circ}$ & $1.412 \mathrm{~m}$ & $1.335 \mathrm{~m}$ & $-0.077 \mathrm{~m}$ \\
R24 & $14.0^{\circ}$ & $142.1^{\circ}$ & $1.570 \mathrm{~m}$ & $1.455 \mathrm{~m}$ & $-0.115 \mathrm{~m}$ \\
\hline \hline
\end{tabular}
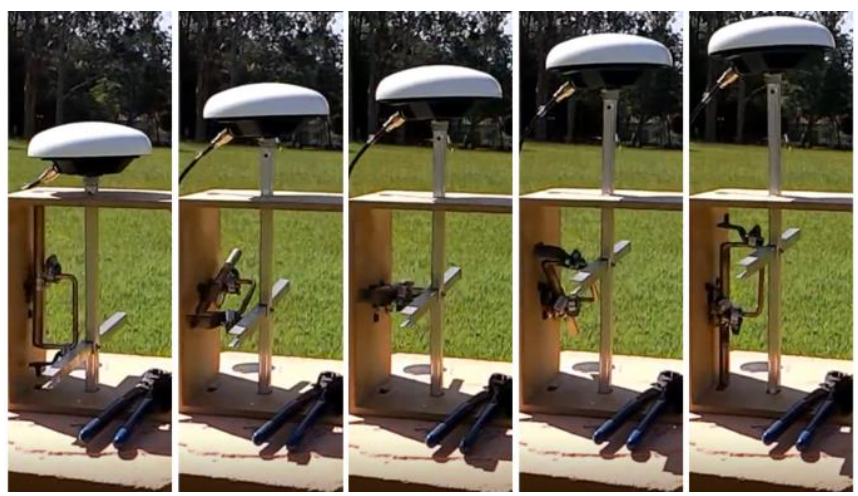

Fig. 1. Photograph time lapse of the antenna vertical displacement device.

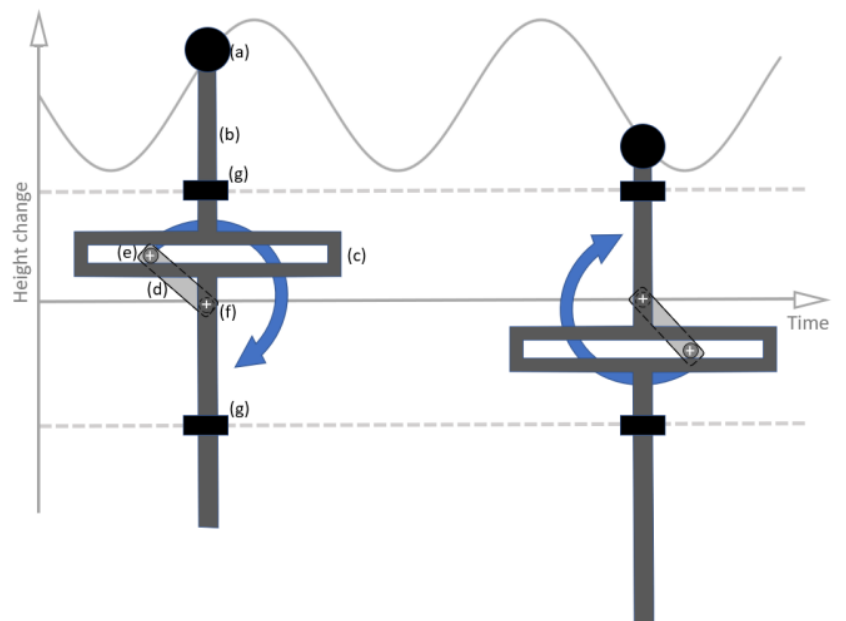

Fig. 2. Diagram of the Scotch yoke device at two times; parts are labeled as: (a) GNSS antenna; (b) connecting rod; (c) sliding yoke; (d) rotating arm; (e) sliding link pin; (f) motor axis; (g) fixed guides. 


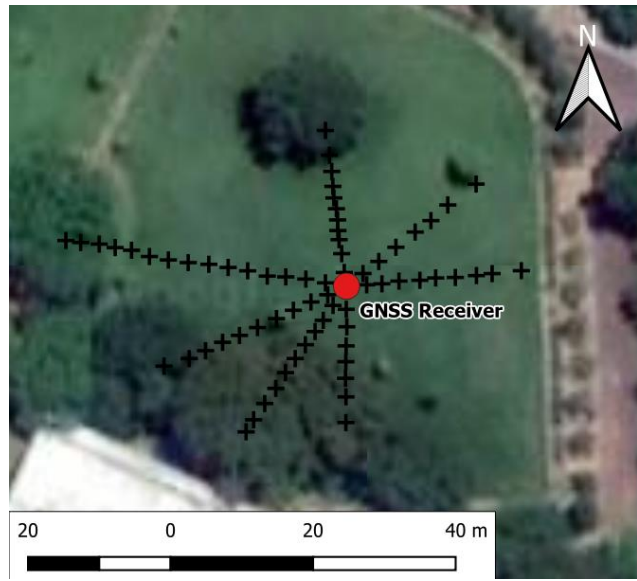

Fig. 3. GNSS antenna location and topography survey points
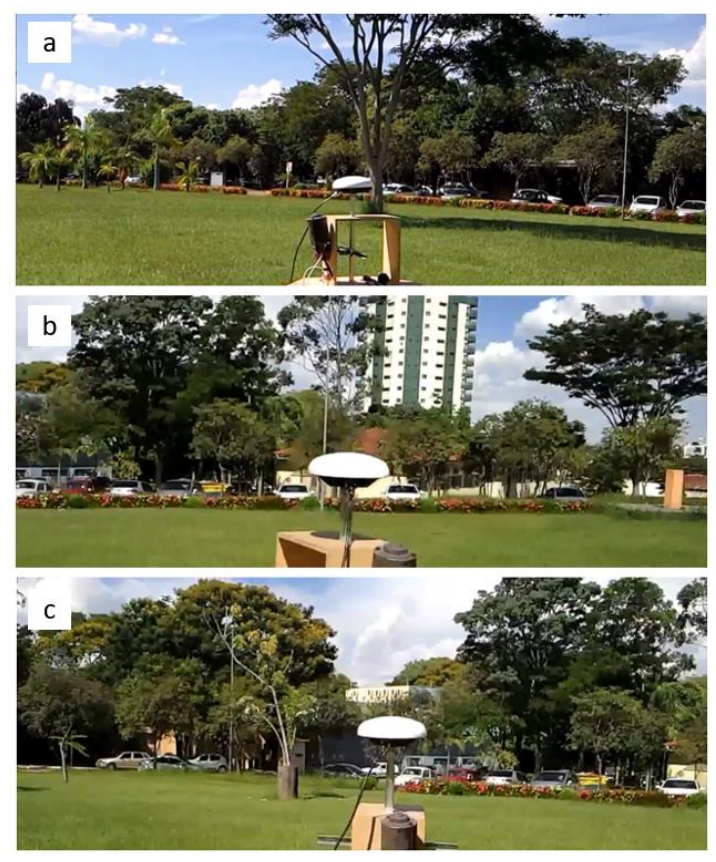

Fig. 4. View of horizon from the antenna site due north (a), east (b), and southeast (c)

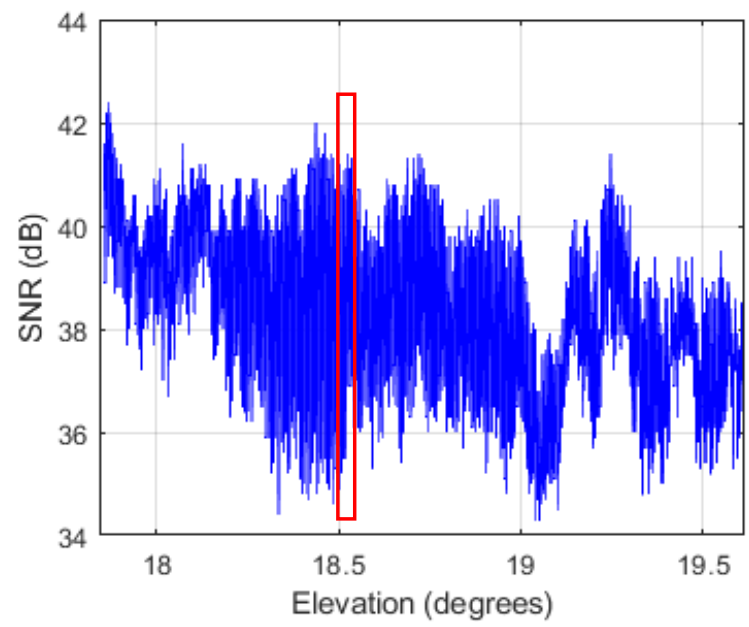

Fig. 5. SNR measurements recorded for satellite R02; the red box is shown with a zoom in Figure 6.

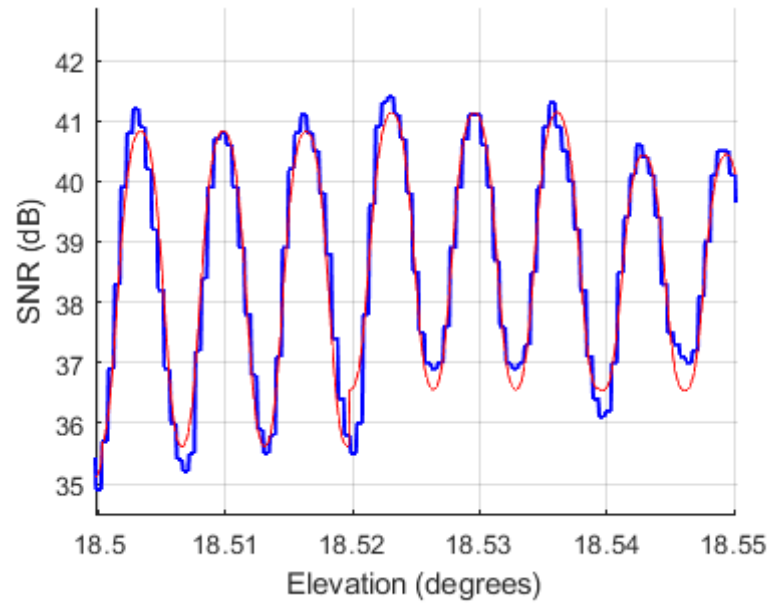

Fig. 6. Zoom on a few sweeping cycles for satellite R02; SNR measurements (blue line) and SNR fitting curve (red line).

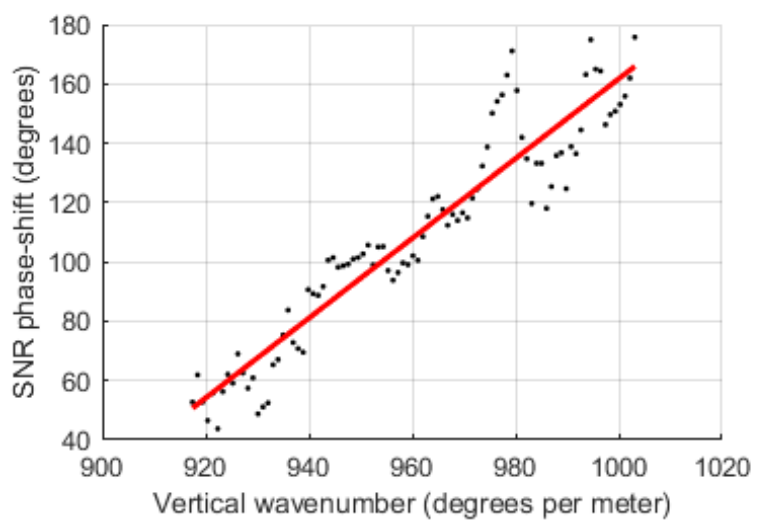

Fig. 7. Static interferometric phase $\Delta \phi$ versus vertical wavenumber $k_{z}$ obtained from SNR fitting for satellite R02 (black dots); simple linear regression fit (red line).

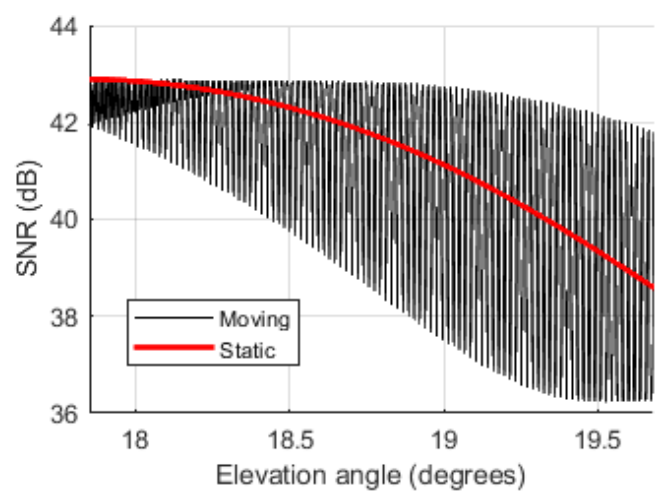

Fig. 8. SNR simulation of a moving antenna (black) and a static antenna (red) with satellite elevation angle corresponding to satellite R02 of the experiment shown in Fig. 5. 